\title{
Fourier Transform Based Transmission Systems for Broadband Wireless Communications
}

\author{
Mingqi Li, Yun Rui and Zhiyong Bu \\ Shanghai Institute of Microsystem and Information Technology, \& Shanghai Advanced \\ Research Institute, Chinese Academy of Sciences, CAS
}

China

\section{Introduction}

In recent years, Fourier Transform (FT), as an effective signal processing technology, is more and more popularly applied to wireless communications. By the FT technologies, it can not only reduce the implementation complexity of traditional transmission systems, but also bring in some new features, thus constructing new transmission systems. The current mainstream transmission schemes utilizing DFT technologies include Orthogonal FrequencyDivision Multiplexing (OFDM) [1], Discrete Fourier Transform Spread Orthogonal Frequency Division Multiplexing (DFT-S-OFDM) [2] and Filter bank modulation [3]. For OFDM systems, by an IDFT at the transmitter, the whole frequency-selective wideband channel is divided into several flat narrow band sub-channels, which is benefit to overcome the effects of multi-path in wireless channels. For DFT-S-OFDM system, the uplink transmission scheme for 3GPP-Long Term Evolution (3GPP-LTE) standard, besides the IDFT served the same function as in the OFDM systems, additional DFT processing is performed to the transmitted constellation symbols before OFDM modulation. In this way, the whole modulation method can be viewed as a DFT-based interpolation processing, and the modulated signals can be regarded as single carrier signals with low Peak-to-Average Power Ratio (PAPR) property. For filter-bank systems, the FT can be used both to reduce implementation complexity and to construct the cyclic prefix $(\mathrm{CP})$ based block transmission scheme, which has merits of both the filter-bank systems with robustness against to multiple access interference (MAI) and the $\mathrm{CP}$ based block transmission systems with simple frequency equalization.

The chapter is organized as follows. Firstly, the implementation structure of OFDM transmitter, time-frequency description of OFDM signals, and effects of timing- and frequency-offset and channel multi-path are discussed detailed. Then, we present DFT-SOFDM system model, describe time-frequency property of DFT-S-OFDM signals, analyze the effects of carrier frequency-offset (CFO) quantitatively and compare the SIR and PAPR performances with that of OFDM systems. Next, a DFT spread Generalized Multi-Carrier (DFT-S-GMC) system is presented. The time-frequency properties of DFT-S-GMC signal, the DFT-based implementation method and the receive SINR are addressed. Finally, conclusions are collected. 


\section{OFDM transmission systems}

OFDM plays a significant role in modern broadband communication systems. The wireline high-speed access technology, i.e. Asymmetric Digital Subscriber Line (ADSL), was the first widely used application for the FT-based OFDM system. Several other wireless standards, such as the IEEE 802.11a Wireless Local Area Network (WLAN) and IEEE 802.16 (WiMAX) series, have adopted OFDM as a key transmission technology. IEEE 802.20 working group on mobile broadband wireless access uses OFDM as the wireless high speed transmission technology. In the area of cellular mobile communications, OFDM was also adopted as a basic downlink transmission scheme of 3GPP-Long-Term Evolution (3GPP-LTE) standard and the incoming 3GPP-LTE-Advanced standard [6]. OFDM is also widely applied in the areas of audio and video broadcasting. Digital Audio Broadcasting (DAB), initiated as a European research project in 1980s, adopts coded OFDM as the transmission technology. DVB-T based on OFDM in an $8 \mathrm{MHz}$ channel is now a popular technology for terrestrial video broadcast in the world. An additional new application area of OFDM is in Ultra-Wideband (UWB) personal area networks [4].

\subsection{OFDM system model}

Figure 1 illustrates the principle structure of OFDM transmitter. Assume that the userspecific $K$ data symbols are $\left\{a_{k}\right\}, 0 \leq k \leq K-1$. After the OFDM modulation, the transmit signals can be expressed as

$$
\begin{gathered}
s_{n}=\frac{1}{\sqrt{N}} \sum_{k=0}^{K-1} a_{k} \exp \left(j 2 \pi n\left(k_{0}+k\right) / N\right), \\
n=-N_{g}, \ldots,-1,0,1, \ldots, N-1
\end{gathered}
$$

where $N_{g}$ is the CP length. To simplify the system model, the localized sub-carrier mapping is applied, i.e., $b_{k^{\prime}}=\left\{\begin{array}{cc}a_{k}, & k^{\prime}=k_{0}+k \\ 0 & \text { otherwise }\end{array}, k^{\prime}=0, \ldots, N-1, k_{0}\right.$ is the user-specific subcarrier allocation offset.

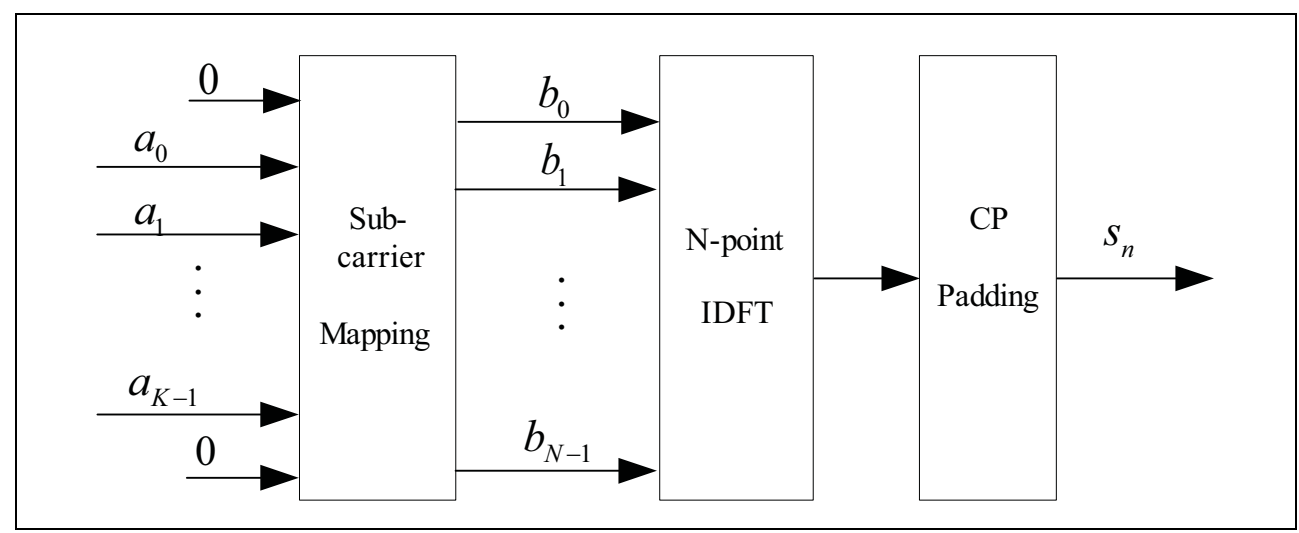

Fig. 1. OFDM transmitter 


\subsection{Time-frequency properties of OFDM signal}

\subsubsection{Time-frequency description of OFDM signal}

Fig. 2 describes the time-frequency property of OFDM signal. According to the implementation principle, OFDM is a block-based transmission scheme. As a result, each OFDM symbol has a rectangle waveform in time-domain. The rectangle waveform can be expressed as

$$
p(t)= \begin{cases}1 & ,|t|<T / 2 \\ 0 & , \text { otherwise }\end{cases}
$$

By the properties of the Fourier transform, the spectrum of each sub-carrier has a sincfunction shape in frequency-domain. Namely, the Fourier transform of $p(t)$ is $P(f)=\operatorname{sinc}(\pi T f) / \pi f$. As it is shown, the spectrum function $P(f)$ has following properties

$$
P(f)= \begin{cases}1 & , f=0 \\ 0 & , f=n / T,(n= \pm 1,2, \ldots)\end{cases}
$$

Equation (3) shows that $P(f), P(f-\Delta f), \ldots, P(f-n \Delta f)$ are orthogonal to each other, and $\Delta f=1 / T$. This means although the spectrum of sub-carriers are overlapped each other, the orthogonality among each sub-carriers can be maintained when the sub-carrier spacing is set to be $\Delta f$.

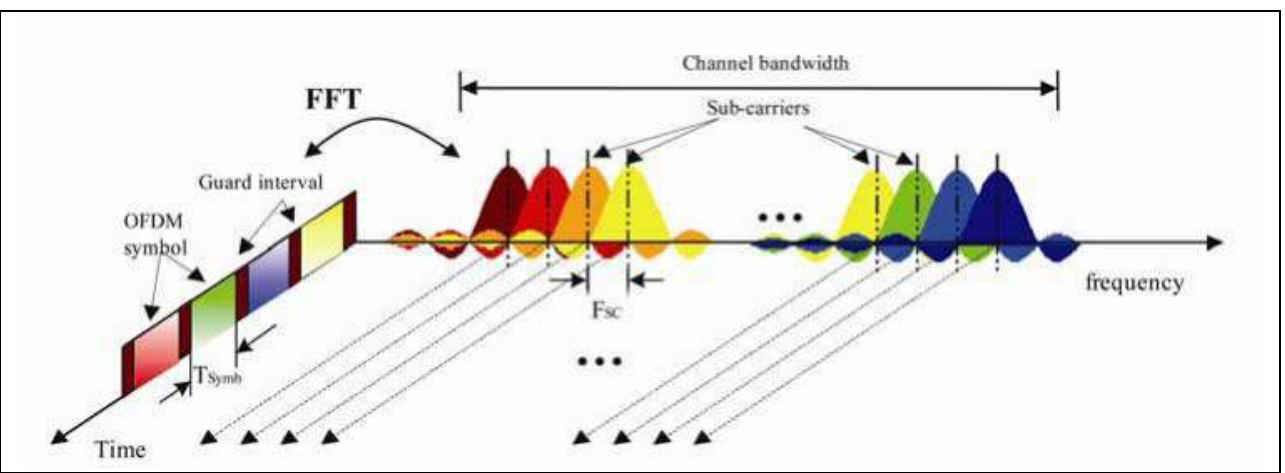

Fig. 2. The time-frequency property of OFDM signal

\subsubsection{Cyclic prefix and frequency-domain equalization}

When the transmitted signal in time domain is $s_{n}$ and the discrete Channel Impulse Response (CIR) is $\left\{h_{i}\right\}_{i=0}^{K-1}$, the received signal can be given as

$$
r_{n}=\sum_{i=0}^{K-1} h_{i} s_{n-i}+w_{n}
$$

where $w_{n}$ is the Additive White Gaussian Noise (AWGN).

According to the DFT theory, the frequency-domain multiplication is equivalent to the timedomain circular-convolution. When the signal $\tilde{s}_{n}, \tilde{s}_{n}=s_{n}, n=0, \ldots, N-1$, is passed through 
the channel, only linear-convolution with the CIR occurs. To mimic circular-convolution, we can copy a partial of samples in the tail of $\tilde{s}_{n}$, and pad to the head of $\tilde{s}_{n}$ to construct a CP. Usually the length of $\mathrm{CP}$ is kept greater or at least equal to the length of the channel delay spread, i.e., $L$. Consequently, by performing DFT to the time-domain signal $r_{n}$, we can get the frequency-domain received signal in the $k$-th sub-carrier

$$
R_{k}=b_{k} H_{k}+W_{k}, k=0, \ldots, N-1
$$

where $H_{k}$ is channel frequency response and $H_{k}=\sum_{i=0}^{L-1} h_{i} e^{j 2 \pi i k / N} . W_{k}$ is the AWGN in frequency domain. $b_{k}$ is the transmitted modulated symbol in the $k$-th sub-carrier as described in Equation (1).

To recover the transmitted symbol, we can apply single-point frequency-domain equalization to each sub-carrier. For example, using ZF equalization, the desired signal can be denoted as

$$
\hat{b}_{k}=\frac{R_{k}}{H_{k}}, k=0, \ldots, N-1
$$

\subsubsection{Effects of time and frequency offset on demodulated signal}

a. Effect of timing offset

Symbol timing is one of the key factors in OFDM synchronization, which decides the accurate selection of FFT window starting position for OFDM demodulation. As shown in Fig. 3, the ideal synchronization position of symbol timing is the first sample of the transmitted signal removing the CP. If the FFT window is opened at the permitted zone, the received signal will produce no Inter-Symbol Interference (ISI), only inducing the common phase rotation on demodulated symbols. While the initial location is selected outside the permitted area, it inevitably will produce the ISI, thus resulting in the Inter-sub-carrier Interference (ICI).

Assuming over the AWGN channel, if the symbol timing position is captured in the permitted zone, then the output demodulation signal on the $k^{\prime}$-th sub-carrier of the $m$-th OFDM symbol is expressed as

$$
\hat{a}_{m, k^{\prime}}=a_{m, k^{\prime}} \exp \left(-j 2 \pi \xi k^{\prime} / N\right), k^{\prime}=0, \ldots, N-1
$$

where $\xi$ is the timing offset. However, if the symbol timing position is outside the permitted zone, for example delay $\xi$ samples, then the output demodulation signal on the $k^{\prime}$-th sub-carrier of the $m$-th OFDM symbol can be denoted as

$$
\begin{aligned}
\hat{a}_{m, k^{\prime}}= & \frac{N-\xi}{N} a_{m, k^{\prime}} \exp \left(j 2 \pi \xi k^{\prime} / N\right) \\
& +\frac{1}{N} \sum_{n=0}^{N-1-\xi} \sum_{k=0 ; k=k^{\prime}}^{K-1} a_{m, k^{\prime}} \exp (j 2 \pi(n+\xi) / N) \\
& +\frac{1}{N} \sum_{n=N-\xi}^{N-1} \sum_{k=0}^{K-1} a_{m+1, k} \exp \left(j 2 \pi\left(n-N-N_{g}+\xi\right) k / N\right) \exp \left(-j 2 \pi n k^{\prime} / N\right)
\end{aligned}
$$


where the second item on the right side is for ICI, and the third part is for ISI. As shown in Fig. 4, the constellations of the demodulated signals are divergent besides the phase rotation.

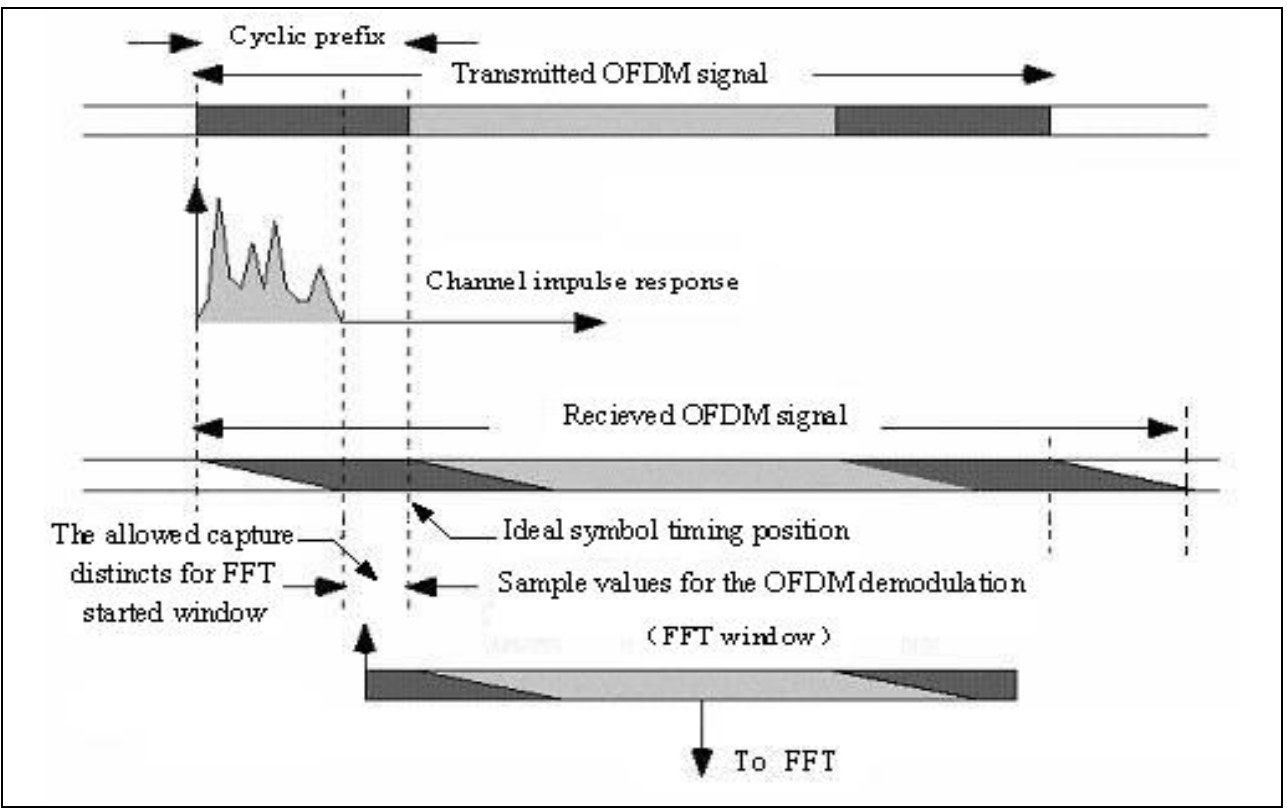

Fig. 3. OFDM symbol timing

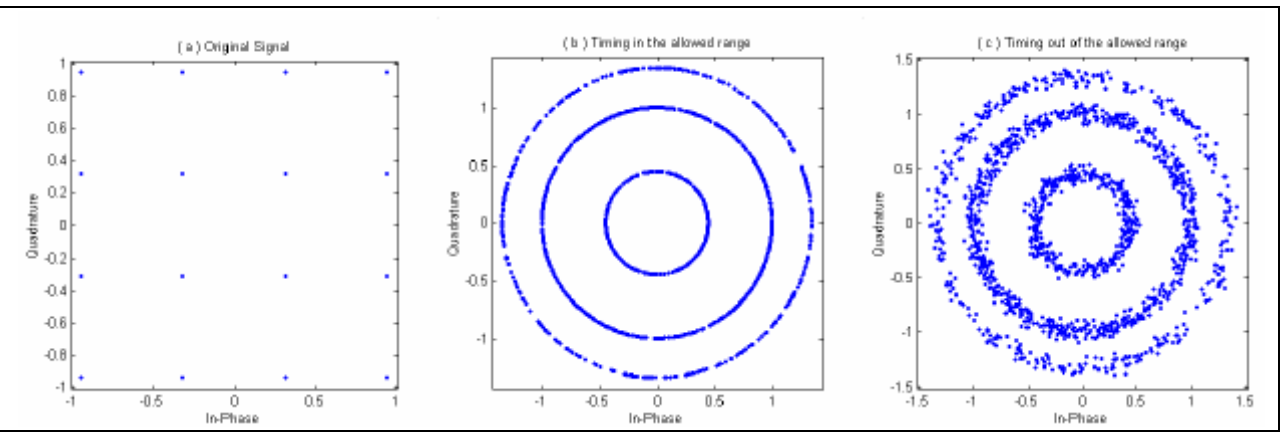

Fig. 4. The constellation of demodulated signal with timing offset

b. Effect of carrier frequency offset

In OFDM system, the existed CFO will lead to frequency shift of the received signal. If the offset of the sub-carrier frequency is integral multiple of sub-carrier spacing, the orthogonality among sub-carriers is still maintained, just with a shift relative to the subcarrier for the data symbols. However, if the frequency offset is a fractional sub-carrier spacing, the ICI will be introduced. For OFDM systems composed of a large number of subcarriers, sub-carrier bandwidth is relatively much smaller compared with the channel 
bandwidth. Therefore, the small amount of frequency offset will result in substantial BER performance degradation.

Assuming the normalized fractional CFO is $\varepsilon$, and then the demodulated signal can be expressed as

$$
a_{k^{\prime}}=\alpha a_{k^{\prime}} \exp (j \pi \varepsilon(N-1) / N)+\sum_{n=0}^{N-1} \sum_{k=0 ; k \neq k^{\prime}}^{K} a_{k^{\prime}} \exp \left(j 2 \pi n\left(k-k^{\prime}+\varepsilon\right) / N\right)
$$

where $\alpha$ is the attenuation experienced by all sub-carriers, and

$$
\alpha=\frac{\operatorname{sinc}(\varepsilon)}{\operatorname{sinc}(\varepsilon / N)}
$$

The second part on the right side of Equation (9) is an ICI item. Fig. 5 shows the demodulated signal constellation under the AWGN channel with $\varepsilon=0.05$. As shown in the figure, the $\mathrm{CFO}$ on one hand results in the whole constellation phase rotation, and on the other hand, due to the impact of ICI, a divergent phenomenon is generated among constellation points.

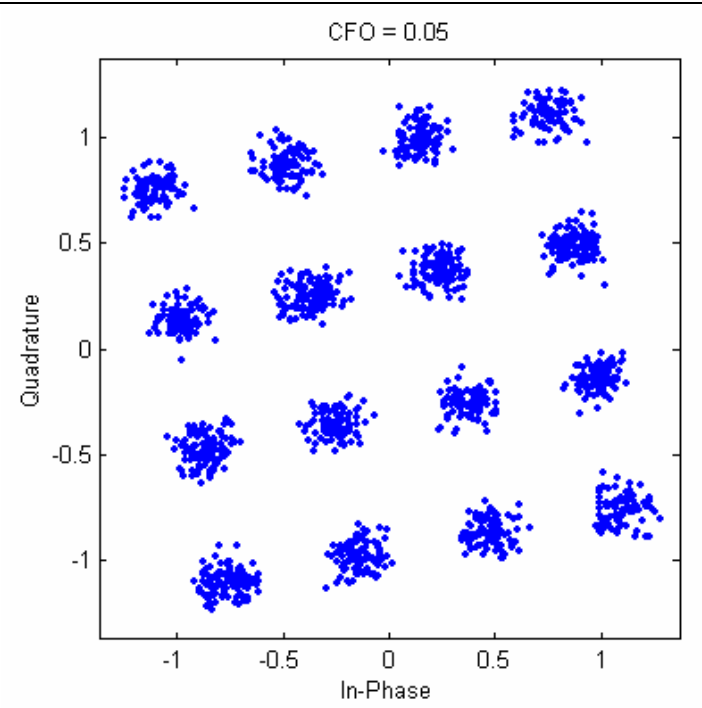

Fig. 5. The constellation of demodulated signal with $\mathrm{CFO}=0.05$

\subsection{Performances analysis and numerical results}

a. SIR effects caused by the carrier frequency-offset

According to Equation (9), the SIR of $k^{\prime}$-th sub-carrier is given as

$$
S I R_{k^{\prime}}^{O}=\frac{|\alpha|^{2}}{E\left[\left|I C I_{k^{\prime}}\right|^{2}\right]}
$$


where $I C I_{k^{\prime}}$ is the inter-sub-carrier interference on the $k^{\prime}$-th sub-carrier, and its variance can be expressed as[15]

$$
E\left[\left|I C I_{k^{\prime}}\right|^{2}\right]=\sum_{\substack{k=0 \\ k \neq k^{\prime}}}^{K-1}\left(\frac{\sin c\left(k-k^{\prime}+\varepsilon\right)}{\sin c\left(\left(k-k^{\prime}+\varepsilon\right) / N\right)}\right)^{2}
$$

\section{b. Uncoded BER Performance of OFDM}

In order to evaluate the error probability, without loss of generality, we focus on the signal received on the first sub-carrier. Moreover, let us now consider the QPSK modulation. By the conditional SINR, the approximate BER becomes [14]

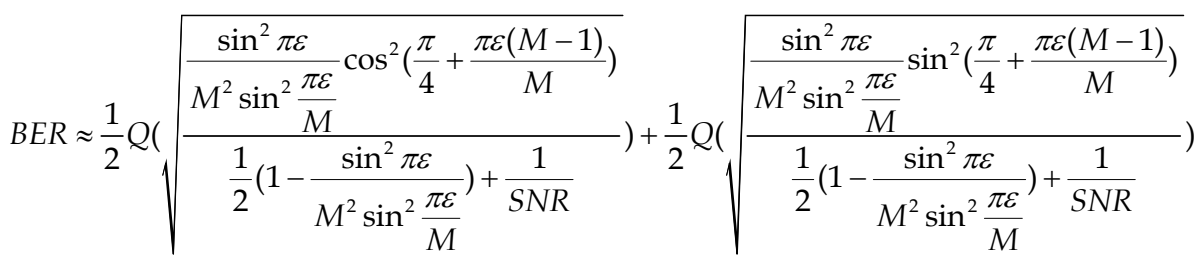

where $M$ is the modulation order, and $S N R$ is the average received signal to noise ratio. Fig. 6 shows simulation results of the impacts of the CFO on the BER performance of OFDM system with QPSK modulation over the AWGN channel.

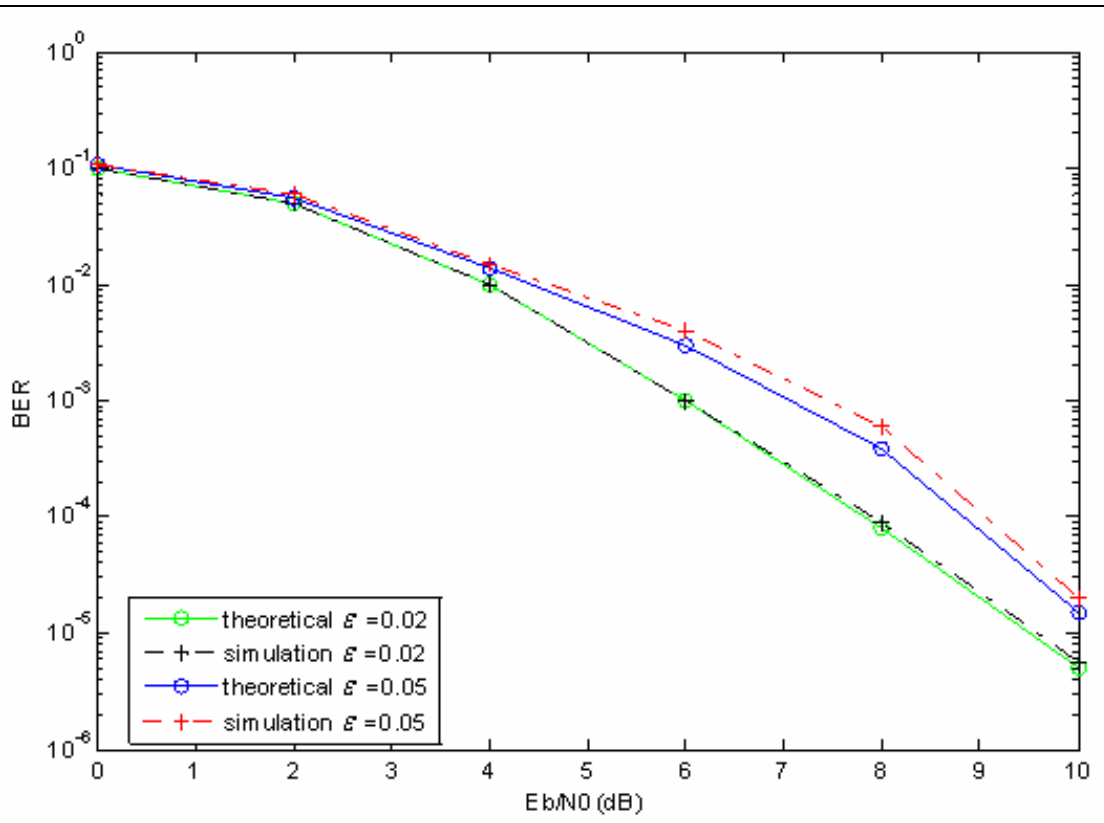

Fig. 6. BER of OFDM system with QPSK modulation under the impacts of the CFO over the AWGN channel 


\section{DFT-S-OFDM transmission systems}

In order to meet the emerged new requirements, many powerful and implementationefficient transmission schemes have been proposed for the standardization of the latest and future communication systems. For the downlink transmission, the OFDM scheme has been widely accepted due to its high spectral efficiency and flexible resource allocation. For the uplink transmission, however, the power efficiency is particularly critical for mobile terminals with the restriction on the transmission power and power consumption. Therefore, the PAPR performance becomes one of the most important criterions in selecting the transmission scheme for the uplink. From this point of view, the single-carrier based frequency division multiple access (SC-FDMA) scheme is favoured by future wideband wireless communications. In fact, one kind of SC-FDMA schemes, i.e., DFT-S-OFDM, is accepted as the uplink basic transmission scheme by the 3GPP-LTE standard and incoming 3GPP-LTE-Advanced standard [5][6]. In order to reduce the PAPR, the DFT-S-OFDM scheme utilizes the DFT spreading processing on the transmitted constellation symbols before OFDM modulation. By this way, the modulation method can be view as a DFT-based interpolation processing, and the modulated signals can be regarded as single carrier signals.

\subsection{DFT-S-OFDM system model}

The structure of DFT-S-OFDM transmitter is shown in Fig. 7. Assume the user-specific $K$ data symbols are $\left\{a_{m}\right\}, 0 \leq m \leq K-1$. After the DFT based spreading, the output signals can be expressed as

$$
x_{k}=\frac{1}{\sqrt{K}} \sum_{m=0}^{K-1} a_{m} \exp (-j 2 \pi m k / K), \quad 0 \leq k \leq K-1
$$

Then according to the localized allocation pattern, the signal is converted into a timedomain signal by $N$-point IDFT processing. $N$ is greater than $K$. After the CP padding, the time-domain signal, i.e., the SC-FDMA symbol, can be written as

$$
s_{n}=\frac{1}{\sqrt{N}} \sum_{k=0}^{K-1} x_{k} \exp \left(j 2 \pi n\left(k_{0}+k\right) / N\right), n=-N_{g}, \ldots,-1,0,1, \ldots, N-1
$$

where $k_{0}$ is the user-specific sub-carrier offset and $N_{g}$ is the CP length.

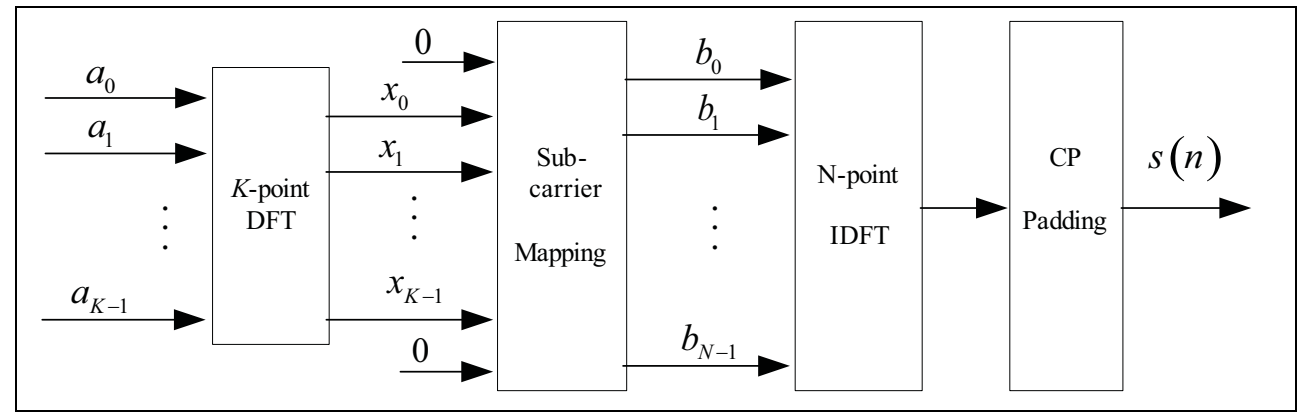

Fig. 7. DFT-S-OFDM transmitter 


\subsection{Time-frequency properties of DFT-S-OFDM signals}

a. Time-frequency description of DFT-S-OFDM signal

Figure 8 describes the time-frequency property of DFT-S-OFDM signal. For DFT-S-OFDM signals, the time-domain waveform can be viewed as a DFT-based interpolation of transmitted constellation symbols. Therefore, the energy distribution within one DFT-SOFDM symbol keeps the same transmission order of the constellation symbols in the timedomain. In the frequency-domain, due to the DFT based spread spectrum processing, the spectrum of each transmitted constellation symbol is distributed on all occupied subcarriers, i.e., each sub-carrier contains only a part of spectrum component of the transmitted constellation symbol, which is substantially different form OFDM signals.

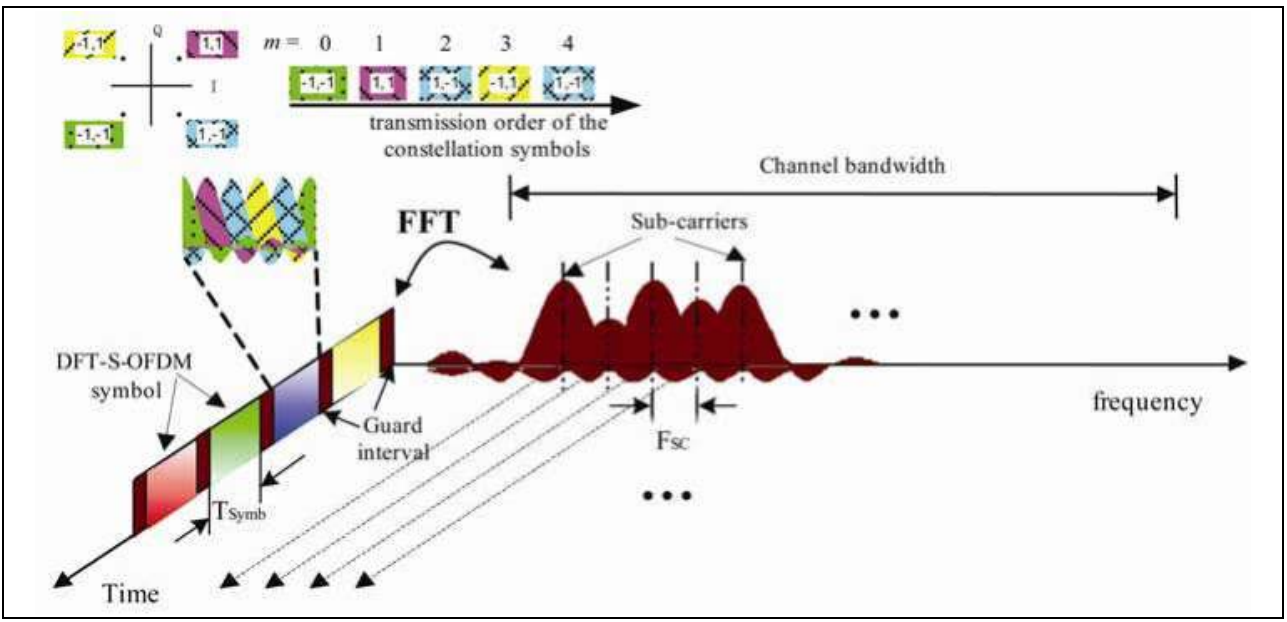

Fig. 8. The time-frequency property of DFT-S-OFDM signal

b. Effects of time and frequency offset on demodulated signal

DFT-S-OFDM systems are subjected to the CFO and TO as well. Although the DFT-S-OFDM scheme is based on the OFDM technology, the effects of CFO on two systems are very different, because the DFT-S-OFDM systems can be viewed as transmitting symbols in the time-domain, whereas the OFDM systems are usually regarded as transmitting symbols in the frequency domain.

1. Effect of time offset

First, if the timing point is allocated inside the permitted zone, similar as the analysis for OFDM system, the received signal in the frequency domain for DFT-S-OFDM can be denoted as

$$
\hat{x}_{k}=x_{k} \exp (-j 2 \pi \xi k / N), \quad k=0,1, \ldots, K-1
$$

While after the K-point IDFT despreading, then the demodulated symbol can be given as

$$
\hat{a}_{m^{\prime}}=\frac{1}{\sqrt{K}} \sum_{k=0}^{K-1} \hat{x}_{k} \exp \left(j 2 \pi m^{\prime} k / K\right), \quad m^{\prime}=0,1, \ldots, K-1
$$

From the above expression, we can see that although the timing point is allocated inside the permitted zone, it not only induce the common phase rotation, but also brings the ICI on the 
demodulated symbols, which is shown in Fig. 9. This character is different from OFDM system. Furthermore, if the timing point is outside the permitted zone, it will cause both the ISI and ICI, which is similar with OFDM.

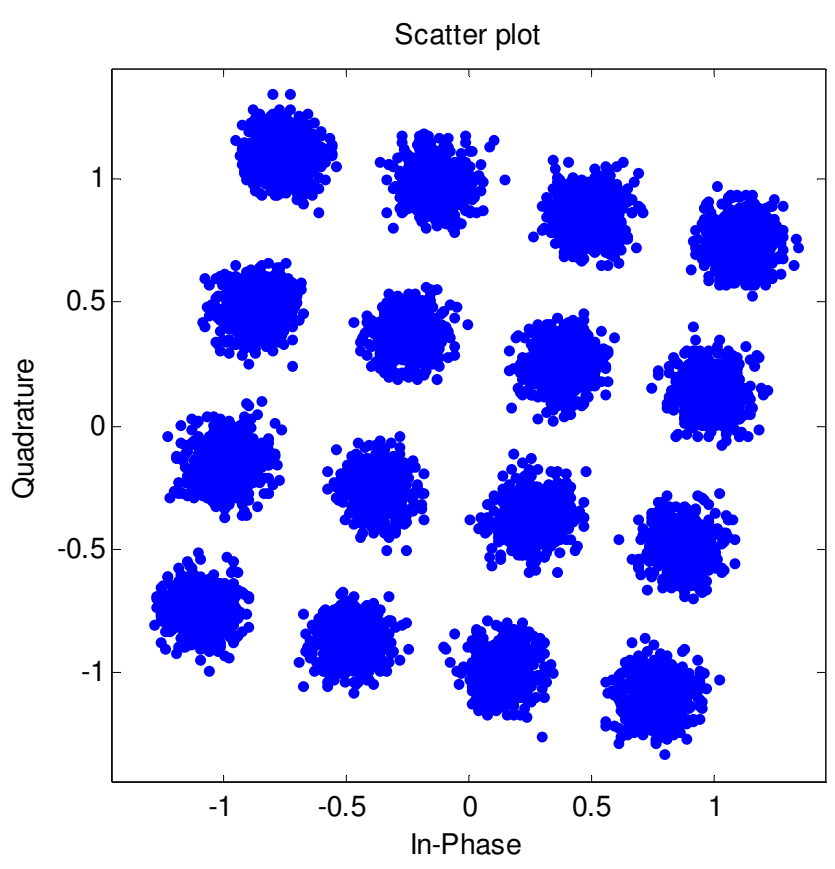

Fig. 9. The constellation of demodulated signal with timing offset

2. Effect of $\mathrm{CFO}$

Considering CFO effects, following CP removing, the received $N$ samples of SC-FDMA symbol for demodulation are

$$
r_{n}=\frac{1}{\sqrt{N}} \sum_{k=0}^{K-1} H_{k+k_{0}} x_{k} \exp \left(j 2 \pi n\left(k_{0}+k+\varepsilon\right) / N\right)+w_{n}, \quad n=0,1, \ldots, N-1
$$

where $w_{n}$ is the complex-valued AWGN on the $n$-th time-domain sample. $H_{k}$ is the channel frequency response (CFR) at the $k$-th sub-carrier, and $H_{k}=\sum_{i=0}^{L-1} h_{i} \cdot \exp \left(-j 2 \pi k \tau_{i} / N\right)$. By DFT and single sub-carrier equalization, the output is

$$
y_{k^{\prime}}=\frac{1}{N} \sum_{n=0}^{N-1} \sum_{k=0}^{K-1} W_{k+k_{0}} H_{k+k_{0}} x_{k} \exp \left(j 2 \pi n\left(k+k_{0}+\varepsilon-k^{\prime}\right) / N\right), \quad k^{\prime}=0,1, \ldots, N-1
$$

where $x_{k}=\frac{1}{\sqrt{K}} \sum_{m=0}^{K-1} a_{m} \exp (-j 2 \pi m k / K), \quad 0 \leq k \leq K-1$.

Then, after the sub-carrier demapping, generally, the $K$ elements extracted from the $N$ sample output of DFT are processed by a $K$-point IDFT, and yields the estimated symbols 


$$
\hat{a}_{m^{\prime}}=\frac{1}{\sqrt{K}} \sum_{k^{\prime}=0}^{K-1} y_{k^{\prime}+k_{0}} \exp \left(j 2 \pi m^{\prime} k^{\prime} / K\right), \quad m^{\prime}=0,1, \ldots, K-1
$$

Because the energy of ICI inducing by CFO is distributed in all the sub-carriers, as shown in Fig.10, the CFO brings about not only the ICI and the linear phase rotation, but also the ISI and the attenuation on the demodulated symbols.

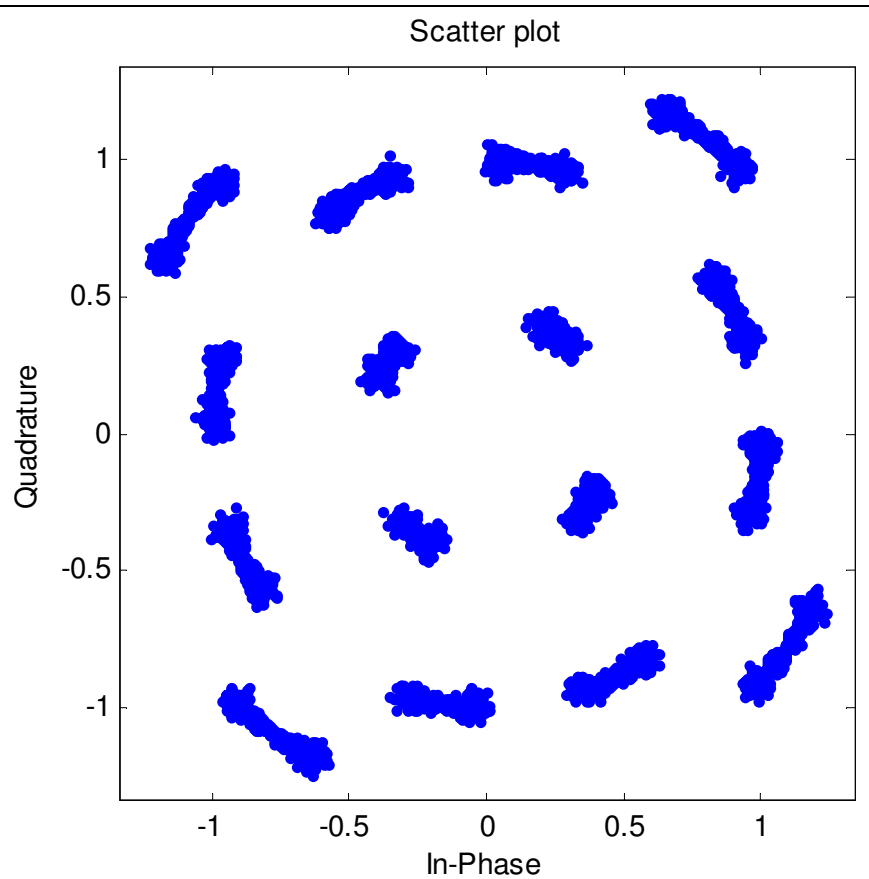

Fig. 10. The constellation of demodulated signal with $\mathrm{CFO}=0.05$

\subsection{Performances analysis and numerical results}

a. SIR effects caused by the carrier frequency-offset

For DFT-S-OFDM systems with CFO and over a flat fading channel, the demodulated symbol can be given as [10]

$$
\hat{a}_{m^{\prime}}=a_{m^{\prime}} \alpha_{m^{\prime}} \exp \left(j 2 \pi m^{\prime} \varepsilon / K\right)+I S I_{m^{\prime}}+I C I_{m^{\prime}}
$$

where $\alpha_{m^{\prime}}, I S I_{m^{\prime}}$ and $I C I_{m^{\prime}}$ are the attenuation term, inter-symbol interference and intersub-carrier interference on the $m^{\prime}$-th demodulated symbol respectively.

$$
\begin{aligned}
\alpha_{m^{\prime}}= & \frac{1}{K} \sum_{k^{\prime}=0}^{N-1} \sum_{k=0}^{K-1} \exp \left(-j 2 \pi\left(k-k^{\prime}+k_{0}+\varepsilon\right) m^{\prime} / K\right) \\
& \cdot \exp \left(j \pi\left(k-k^{\prime}+k_{0}+\varepsilon\right)(N-1) / N\right) \frac{\sin c\left(k-k^{\prime}+k_{0}+\varepsilon\right)}{\sin c\left(\left(k-k^{\prime}+k_{0}+\varepsilon\right) / N\right)}
\end{aligned}
$$




$$
\begin{aligned}
I S I_{m^{\prime}}= & \frac{1}{K} \sum_{k^{\prime}=0}^{N-1} \sum_{k=0}^{K-1} \sum_{\substack{m=0 \\
m \neq m^{\prime}}}^{K-1} a_{m} \exp \left(-j 2 \pi\left(m k-m^{\prime} k^{\prime}+m^{\prime} k_{0}\right) / K\right) \\
& \cdot \exp \left(j \pi\left(k-k^{\prime}+k_{0}+\varepsilon\right)(N-1) / N\right) \frac{\sin c\left(k-k^{\prime}+k_{0}+\varepsilon\right)}{\sin c\left(\left(k-k^{\prime}+k_{0}+\varepsilon\right) / N\right)} \\
I C I_{m^{\prime}} & =\frac{1}{N K} \sum_{k^{\prime}=K}^{N-1} \sum_{n=0}^{N-1} \sum_{k=0}^{K-1} \sum_{m=0}^{K-1} a_{m} \exp (-j 2 \pi m k / K) \exp \left(j 2 \pi n\left(k+k_{0}\right) / N\right) \\
& \cdot \exp (j 2 \pi \varepsilon n / N) \exp \left(-j 2 \pi n k^{\prime} / N\right) \exp \left(j 2 \pi m^{\prime}\left(k^{\prime}-k_{0}\right) / K\right)
\end{aligned}
$$

The SIR of the $m^{\prime}$-th demodulated symbol is

$$
S I R_{m^{\prime}}^{(2)}=\frac{\left|\alpha_{m^{\prime}}\right|^{2}}{E\left[\left|I S I_{m^{\prime}}+I C I_{m^{\prime}}\right|^{2}\right]}
$$

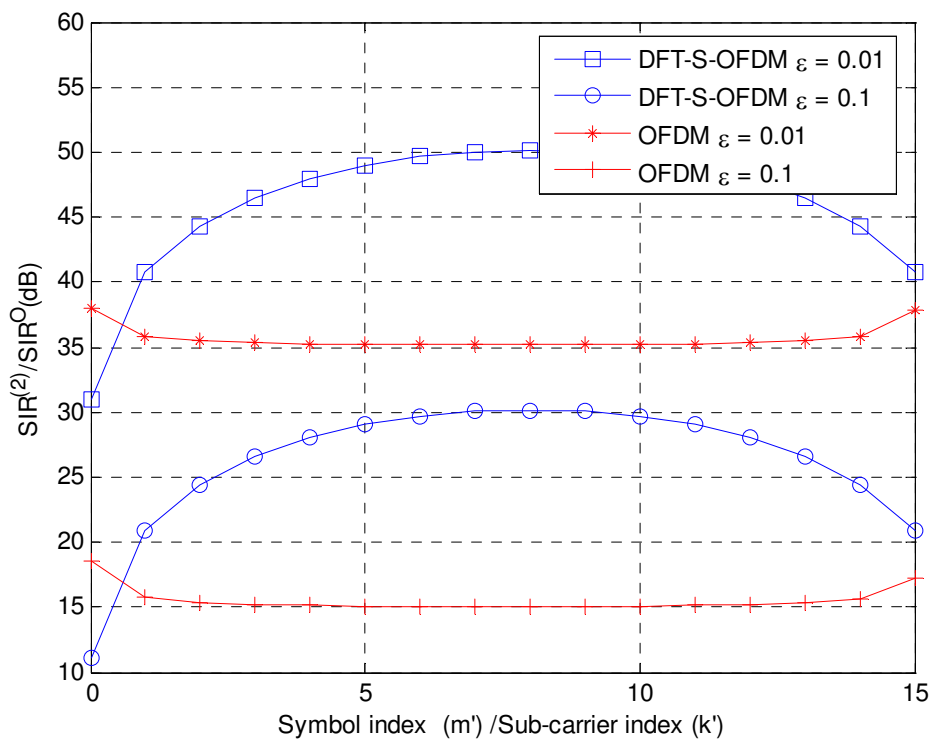

Fig. 11. SIR comparison between DFT-S-OFDM and OFDM system with CFO

As shown in the figure, except the first symbol, all other demodulated symbols of DFT-SOFDM system have a much higher SIR than that of OFDM system under the same CFO condition.

b. Uncoded BER Performance of CFO Effect [16]

We first derive an exact closed-form BER expression for the DFT-S-OFDM system without channel coding. As we know, an arbitrary rectangular QAM can be viewed as two independent pulse amplitude modulation (PAM), i.e., $I$-ary and $J$-ary PAM's, through two 
quadrature branches. As a result, the average bit probability of the detected symbol $\tilde{a}_{m^{\prime}, j}$ in the presence of $\mathrm{CFO}$ can be obtained by averaging the bit error probabilities from [7]

$$
P_{m^{\prime}}^{j}=\frac{1}{\log _{2}(I \cdot J)}\left(\sum_{k=1}^{\log _{2} I} P_{I}(k)+\sum_{l=1}^{\log _{2} J} P_{J}(l)\right)
$$

where

$$
\begin{gathered}
P_{I}(k)=\frac{1}{I} \sum_{i=0}^{\left(1-2^{-k}\right) I-1}(-1)^{\left\lfloor\frac{i \cdot 2^{k-1}}{I}\right\rfloor\left[2^{k-1}-\left\lfloor\frac{i \cdot 2^{k-1}}{I}+\frac{1}{2}\right\rfloor\right]} \\
\cdot \operatorname{erfc}\left((2 i+1) \sqrt{\frac{3 \log _{2}(I \cdot J) S I N R_{m}^{j}}{\left(I^{2}+J^{2}-2\right) M}}\right)
\end{gathered}
$$

where $M$ is the number of bits for the special modulated symbol, and $S I N R_{m}^{j}$ denotes the SINR per modulated symbol, which can be obtained from the above subsection. In addition, $\operatorname{erfc}(\cdot)$ is the complementary error function, and $\lfloor x\rfloor$ denotes the largest integer to $x$. Similarly, the $P_{J}(l)$ can be denoted as the above. Note that for $I=2$ and $J=1$, equation (26) reduces to the BER of a BPSK signal.

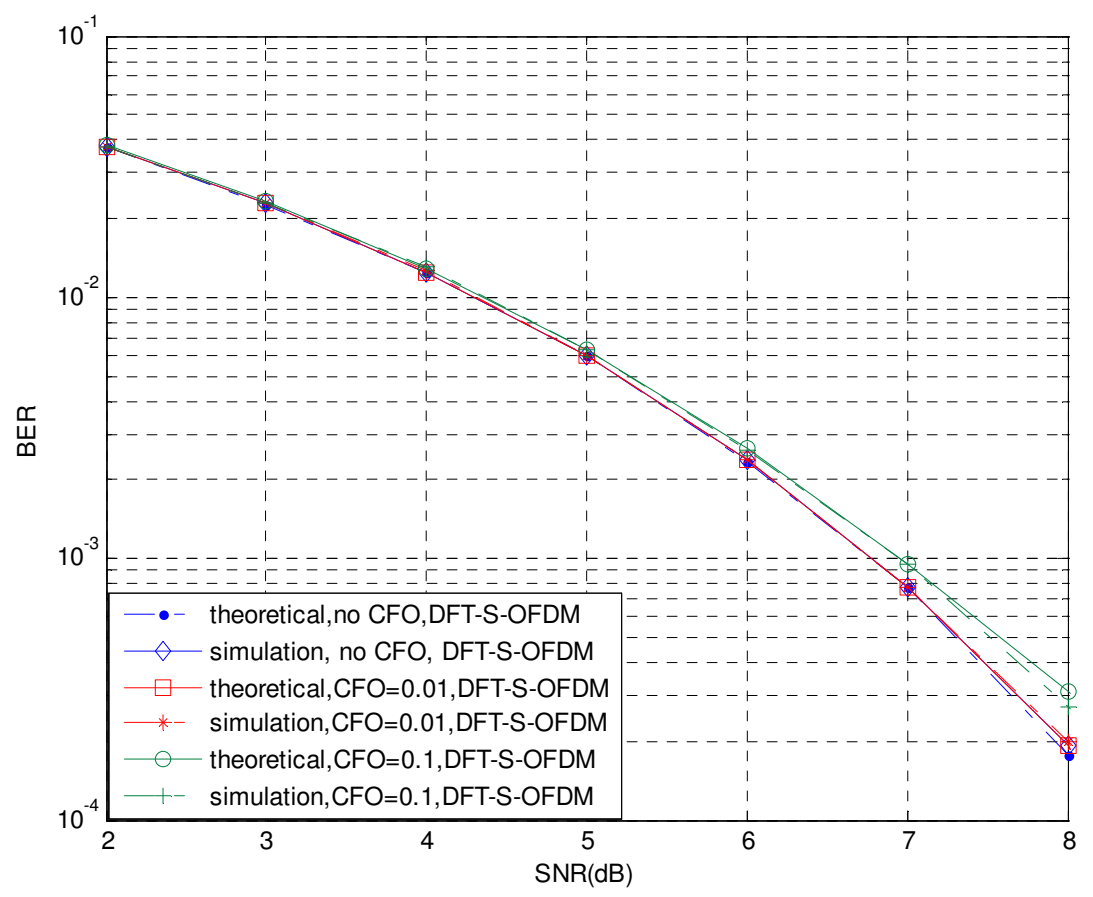

Fig. 12. BER comparison of the CFO effect for uncoded DFT-S-OFDM systems 
Therefore, based on the derived SINR for the demodulated symbol in the above section, the closed-form BER expression in the presence of CFO for DFT-S-OFDM system can be given as

$$
P_{D F T \text {-S-OFDM }}^{\text {uncoded }}=\frac{1}{Q} \sum_{j=0}^{N-1} \sum_{m^{\prime}=0}^{D-1} P_{m^{\prime}}^{j}
$$

Then, using the average BER of the DFT-S-OFDM system, we can compute the effective SINR by the mapping function as shown in (29). For the individual modulation, such as BPSK, the effective SINR can be denoted as

$$
\operatorname{SINR}_{\text {eff }}^{\text {uncoded }}=\left[\operatorname{erfc}^{-1}\left(P_{D F T-S-O F D M}^{\text {uncoded }}\right)\right]^{2}
$$

As a result, the SNR degradation for DFT-S-OFDM system can be presented as

$$
D_{\text {DFT-S-OFDM }}=10 \log _{10} \frac{E_{s}}{\sigma^{2} \cdot S I N R_{\text {eff }}^{\text {uncoded }}}
$$

As can be seen from the figure, the theoretical result using the exact expression agrees well with the simulation results. This clearly shows that the exact expression for calculating the effective SINR in equation (29) can be used in order to assess the effect of the carrier frequency offset accurately.

c. Turbo coded BER Performance of CFO Effect

In this section, we will focus on the BER performance in the presence of CFO for the coded DFT-S-OFDM system. Due to the mathematical complexity of the iterative turbo decoding algorithm, the analytical derivation of the BER of turbo codes is not available. To simplify the analysis, the BER of coded system in AWGN channels can be approximated by an expression of the form [8]

$$
P_{D F T-S-O F D M}^{\text {Coded }}=\exp \left(-\frac{\gamma}{\beta}\right)
$$

where $\gamma$ is the received effective SINR. Parameters $\beta$ is mode-dependent, and can be obtained by fitting the curves to the exact simulated BER. As a result, the exponential effective SINR mapping (EESM) method is considered to incorporate the SINR of all the detected symbols. The formulation of the incorporated SINR can be expressed as [9]

$$
S I N R_{\text {eff }}^{\text {coded }}=-\beta \ln \left(\frac{1}{D} \sum_{j=0}^{N-1} \sum_{m^{\prime}=0}^{D-1} \exp \left\{-\frac{S I N R_{m^{\prime}}^{j}}{\beta}\right\}\right)
$$

Similar to (30), the SNR degradation can be also obtained.

Fig.13 shows the BER performance in DFT-S-OFDM systems for turbo coded modulation, where the QPSK modulation and 1/2 coding rate is assumed. In addition, the EESM method in (32) is used to incorporate the SINR of all the detected symbols, which is different in the presence of $\mathrm{CFO}$, and $\beta$ is achieved through the simulation. As we can see from the figure, the BER mapping curve for CFO $\varepsilon=0.01$ and $\varepsilon=0.1$ is very close to that without CFO over the equivalent SINR. 


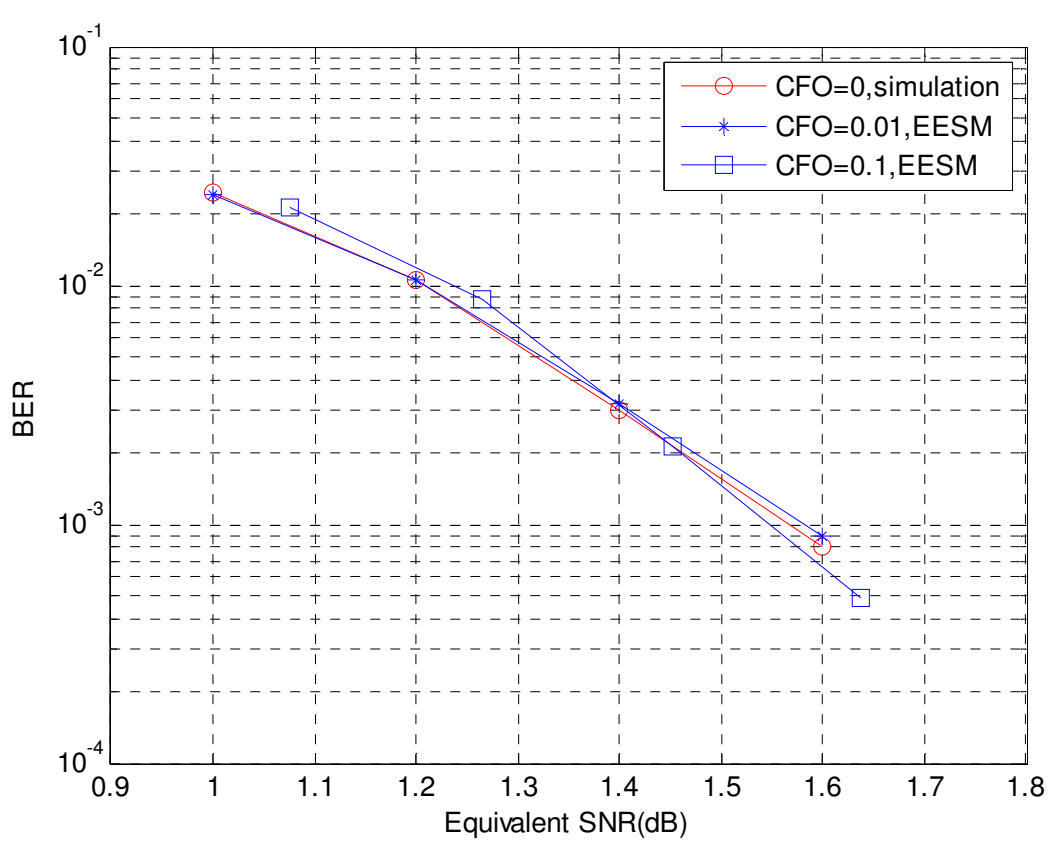

Fig. 13. BER performance with and without the CFO effect

d. Post-processing SINR of DFT-S-OFDM [11]

For DFT-S-OFDM system, the transmit signal vector without $\mathrm{CP}$ can be given as

$$
S=F_{N}^{H} T_{N, M}^{m} F_{M} D
$$

where $T_{N, M}^{m}$ is the mapping matrix for sub-carrier assignment, $F_{M}$ is the M point FFT matrix and $F_{N}^{H}$ is the N point IFFT matrix, $D=\left[\begin{array}{lll}d_{1} & \cdots & d_{M}\end{array}\right]^{T}$ is the data vector.

Then, at the receiver, the vector of detection metric after FDE is then given as

$$
\begin{aligned}
\widehat{D} & =F_{M}^{H} T_{N, M}^{m}{ }^{H} H^{H} W\left(H T_{N, M}^{m} F_{M} D+Z\right) \\
& =F_{M}^{H} \widetilde{H}_{W, M}^{m} F_{M} D+F_{M}^{H} \bar{H}_{W, M}^{m} Z_{M}
\end{aligned}
$$

where $W=\operatorname{diag}\left\{\omega_{1}, \omega_{2}, \cdots, \omega_{N}\right\}$ is the frequency domain equalizer, and

$$
\begin{gathered}
\tilde{H}_{W, M}^{m}=\operatorname{diag}\left\{\left|H_{m}\right|^{2} \omega_{m},\left|H_{m+1}\right|^{2} \omega_{m+1}, \ldots,\left|H_{m+(M-1)}\right|^{2} \omega_{m+(M-1)}\right\} \\
\bar{H}_{W, M}^{m}=\operatorname{diag}\left\{H_{m}^{*} \omega_{m}, H_{m+1}^{*} \omega_{m+1}, \ldots, H_{m+(M-1)}^{*} \omega_{m+(M-1)}\right\}
\end{gathered}
$$

Hence, the SINR for DFT-S-OFDM with FDE is given as 


$$
\operatorname{SINR}=\frac{\left.\left.\left|\frac{1}{M} \sum_{k=1}^{M-1}\right| H_{m+k}\right|^{2} \omega_{m+k}\right|^{2}}{\frac{1}{M} \sum_{k=1}^{M-1}\left|H_{m+k}\right|^{2}\left|\omega_{m+k}\right|^{2} \sigma^{2}+\frac{1}{M} \sum_{k=1}^{M-1}\left|H_{m+k}\right|^{4}\left|\omega_{m+k}\right|^{2}-\left.\left.\left|\frac{1}{M} \sum_{k=1}^{M-1}\right| H_{m+k}\right|^{2} \omega_{m+k}\right|^{2}}
$$

Furthermore, for ZF and MMES equalizer, the SINR expression can be simplified respectively as

$$
\operatorname{SINR}=\left[\frac{1}{M} \sum_{k=0}^{M-1} \frac{\sigma^{2}}{\left|H_{m+k}\right|^{2}}\right]^{-1}
$$

and

$$
\operatorname{SINR}=\left[\frac{1}{\frac{1}{M} \sum_{k=0}^{M-1} \frac{\left|H_{m+k}\right|^{2}}{\left|H_{m+k}\right|^{2}+\sigma^{2}}}-1\right]^{-1}
$$

\section{DFT-S-GMC transmission systems}

\subsection{DFT-S-GMC system model}

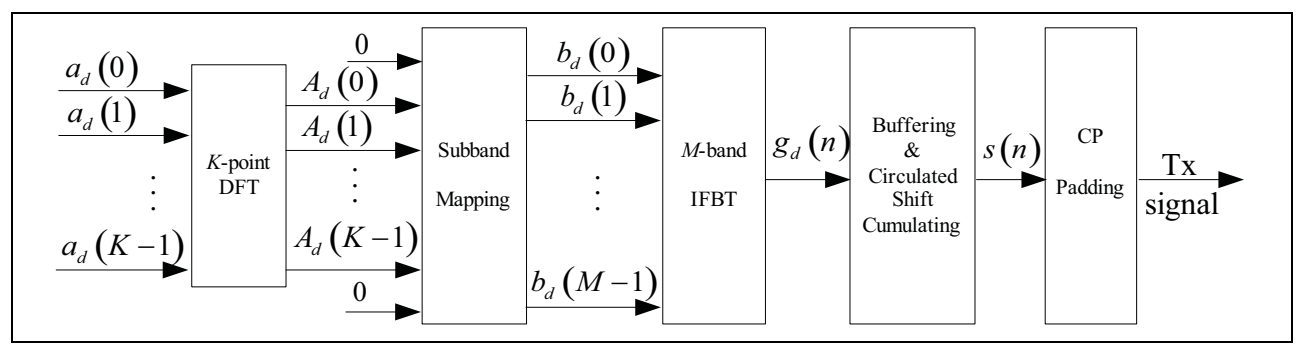

Fig. 14. DFT-S-GMC transmitter

The structure of the DFT-S-GMC transmitter is illustrated in Fig. 14 [12]. Assume that the input parallel modulated constellation symbol sequence is $\left\{a_{d}(k)\right\}, 0 \leq k \leq K-1$ and $0 \leq d \leq D-1$. Note that $K$ is the number of the user-specific occupied sub-bands, and $D$ is the number of inverse filter-bank transform (IFBT) symbols transmitted during each DFT-SGMC symbol.

The input data sequence is passed through K-point DFT for spectrum spreading, yields the output signal

$$
A_{d}\left(k^{\prime}\right)=\frac{1}{\sqrt{K}} \sum_{k=0}^{K-1} a_{d}(k) \exp \left(-j 2 \pi k k^{\prime} / K\right), \quad 0 \leq k^{\prime} \leq K-1
$$

Then, the signals are sent to the sub-band mapping module, yields 


$$
b_{d}(m)=\left\{\begin{array}{cc}
A_{d}\left(k^{\prime}\right), & m=k_{0}+k^{\prime} R \\
0, & m \neq k_{0}+k^{\prime} R
\end{array}, \quad 0 \leq m \leq M-1\right.
$$

where $M$ is the total number of sub-bands, $k_{0}$ is the user-specific sub-band offset, $R$ is the repetition factor. For DFT-S-GMC system, both distributed and localized mapping policy could be supported, which is corresponding to $\mathrm{R}$ greater than or equal to one respectively. After sub-bands mapping, an IFBT is performed on the data sequence, yields the output signal, i.e., the IFBT symbol, as

$$
\begin{aligned}
g_{d}(n)= & \sum_{m=0}^{M-1} b_{d}(m) \exp (j 2 \pi m n / M) f_{p}(n), \\
& 0 \leq n \leq L-1
\end{aligned}
$$

where $f_{p}(n)$ is the impulse response of the prototype filter for the filter bank, and can be set to a Square Root Raised Cosine (SRRC) function. The prototype filter is with a normalized energy, i.e. $\sum_{n=0}^{L-1}\left|f_{p}(n)\right|^{2}=1$ and a length $L$ which is integer times of $M$. Then, each IFBT symbol is zero-padded to form a $Q$-sample data block

$$
\tilde{g}_{d}(n)=\left\{\begin{array}{cc}
g_{d}(n), & 0 \leq n \leq L-1 \\
0, & L \leq n \leq Q-1
\end{array}\right.
$$

where $Q=D \times N$.

Next, $D$ consecutive Q-sample data blocks will be processed by buffering and cyclic-shift cumulating with shift interval $N$, and the output can be described as

$$
s(n)=\sum_{d=0}^{D-1} \tilde{g}_{d}((n-d N))_{Q}, 0 \leq n \leq Q-1
$$

where $((\cdot))_{Q}$ denotes modulus operation.

\subsection{Time-frequency properties of DFT-S-GMC signals}

From equation (44), the DFT-S-GMC symbol is formed by cyclically accumulating several IFBT symbols in time domain, and each IFBT symbol has a SRRC waveform. Therefore, as shown in Fig. 15, the spectrum of each sub-band has a Raised Cosine function shape in frequency-domain. Moreover, the sub-band spacing is specially designed and differs from any conventional filter-bank systems in that a certain guard band is inserted between neighbouring sub-bands. By adding some guard band between the sub-bands, the nearorthogonality between neighbouring sub-bands is guaranteed, which further simplifies the detection algorithm greatly. Meanwhile, interferences among successive symbols for each sub-band could be easily mitigated in the receiver because of the shift orthogonality of prototype filter and the narrow-band single carrier transmission. Similar to DFT-S-OFDM systems, DFT-S-GMC systems also exploit DFT based spreading among sub-bands. Therefore, each sub-band contains only a part of spectrum component of transmitted constellation symbols, and transmitted signal over all occupied sub-bands can be viewed as single-carrier signal as a whole. 


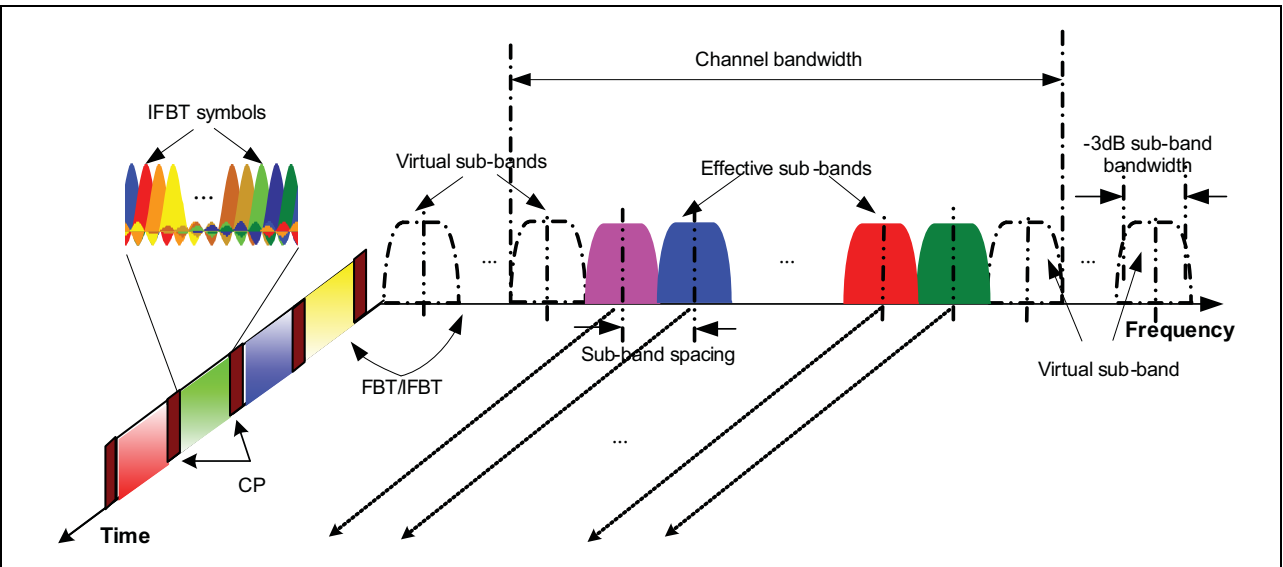

Fig. 15. The time-frequency property of DFT-S-GMC signal

\subsection{Frequency-domain implementation structure}

a. The frequency-domain equivalent implementation of the DFT-S-GMC transmitter As described in equation (44), it can be seen that transmitted symbols are multiplexed within each DFT-S-GMC symbol by both time and frequency dimensions. Taking Q-point FFT on the DFT-S-GMC modulation signal, the output signal can be expressed as

$$
S(q)=\frac{1}{\sqrt{Q}} \sum_{t=0}^{Q-1} \sum_{n=0}^{D-1} g_{((t-n N))_{Q}}(n) \exp (-j 2 \pi q t / Q)
$$

Since

$$
\begin{gathered}
\frac{1}{\sqrt{Q}} \sum_{t=0}^{Q-1} g_{((t-n N))_{Q}}(n) \exp (-j 2 \pi q t / Q) \\
=\frac{1}{\sqrt{Q}} \sum_{t=0}^{Q-1} g_{t}(n) \exp (-j 2 \pi q t / Q) \exp (-j 2 \pi q n / D) \\
\frac{1}{\sqrt{Q}} \sum_{t=0}^{Q-1} g_{t}(n) \exp (-j 2 \pi q t / Q) \\
=\sum_{m=0}^{M-1} b_{m}(n) \frac{1}{\sqrt{Q}} \sum_{t=0}^{Q-1} f_{p}(t) \exp (j 2 \pi m t / M) \exp (-j 2 \pi q t / Q) \\
=\sum_{m=0}^{M-1} b_{m}(n) F_{m}(q)
\end{gathered}
$$

where

$$
\begin{gathered}
F_{m}(q)=\frac{1}{\sqrt{Q}} \sum_{t=0}^{Q-1} f_{p}(t) \exp (j 2 \pi m t / M) \exp (-j 2 \pi q t / Q) \\
, \quad 0 \leq q \leq Q-1 ; \quad 0 \leq m \leq M-1
\end{gathered}
$$




$$
S(q)=\sum_{m=0}^{M-1} F_{m}(q) \sum_{n=0}^{D-1} b_{m}(n) \exp (-j 2 \pi q n / D)
$$

In fact, $F_{m}(q)$ is the frequency response of the prototype filter for $m$-th sub-band. Therefore, the DFT-S-GMC modulation signal can be expressed as

$$
\begin{aligned}
& s(t)=\frac{1}{\sqrt{Q}} \sum_{q=0}^{Q-1} S(q) \exp (j 2 \pi q t / Q) \\
& =\frac{1}{\sqrt{Q}} \sum_{q=0}^{Q-1} \sum_{m=0}^{M-1} F_{m}(q) \underbrace{\sum_{n=0}^{D-1} b_{m}(n) \exp (-j 2 \pi q n / D)}_{\text {Cyclic extended D-point DFT }} \exp (j 2 \pi q t / Q) \\
& \text { Weighting in frequency-domain } \\
& \text { multi-sub-bands summation } \\
& \text { Q-point IDFT }
\end{aligned}
$$

From equation (50), the frequency-domain implementation structure of DFT-S-GMC transmitter can be given by Fig. 16.

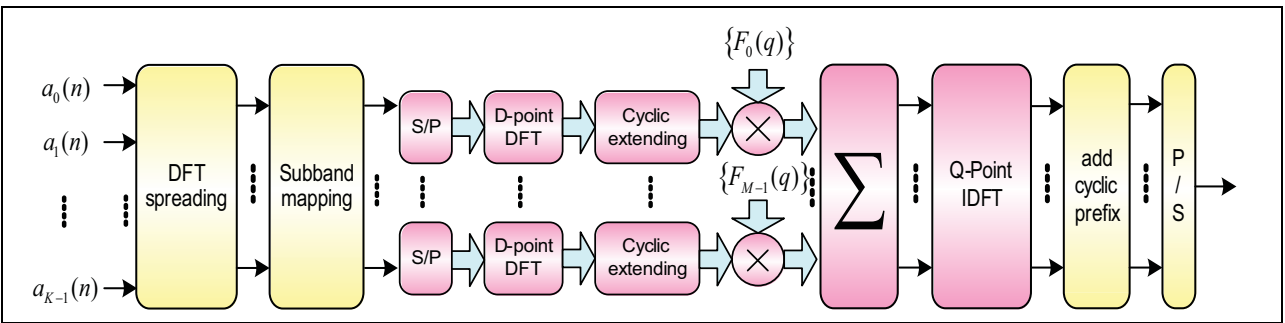

Fig. 16. Frequency-domain implementation structure of DFT-S-GMC transmitter

b. The simplified frequency-domain equivalent implementation of the DFT-S-GMC transceiver

From equation (49), the frequency response of the prototype filter for each sub-band has the energy over all $Q$ tones. However, in fact, the most energy of the frequency response is just over several tones. Therefore, by selecting a set of values with energy greater then a proportional of the total energy of $F_{m}(q), F_{m}(q)$ can be simplified as

$$
\tilde{F}_{m}(q)=\left\{\begin{array}{cc}
F_{m}(q), \quad \sum_{q \in \Omega}\left|F_{m}(q)\right|^{2}>\xi \sum_{q=0}^{Q-1}\left|F_{m}(q)\right|^{2}, \quad q \in \Omega \\
0, \quad \text { otherwise }
\end{array}\right.
$$

With proper designed prototype filter, the frequency response of the prototype filter for each sub-band can be simplified such that the number of total selected values for all subbands is equal to the number of total tones $Q$. By this way, the signals for each sub-band can be even mapped to the tones directly and exclusively, and the simplified implementation structure is shown in Fig.17.

As shown in Fig. 17, by tone mapping, rather than summation processing, the implementation complexity of DFT-S-GMC transmitter can be significantly reduced, and the performance loss is very limited as illustrated by the following simulation results. 


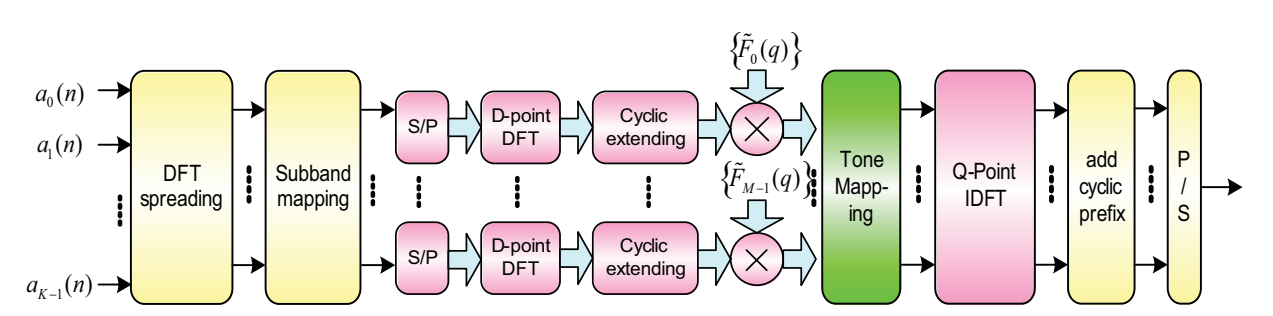

Fig. 17. Simplified implementation structure of DFT-S-GMC transmitter

\subsection{Performances analysis and numerical results}

a. Post-processing SINR with frequency-domain equalization

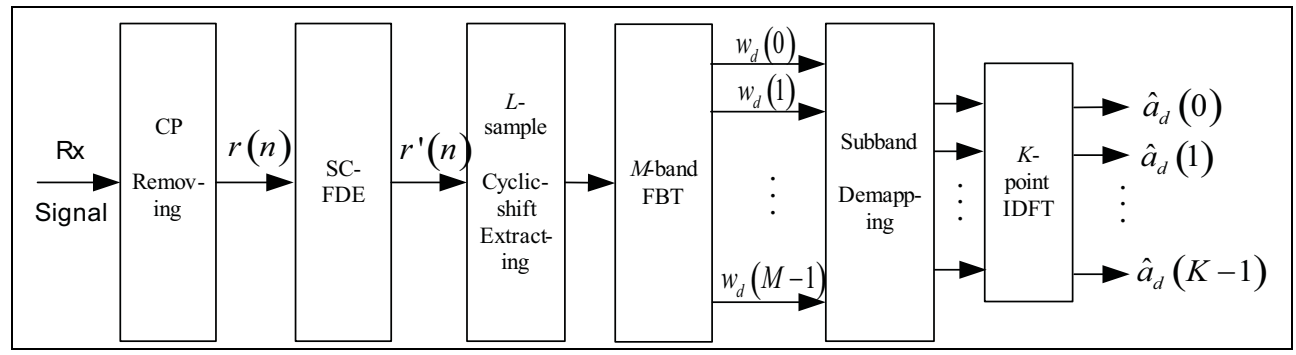

Fig. 18. DFT-S-GMC receiver

At the receiver side, shown in Fig. 18, after removing the $\mathrm{CP}$, and going through the Q-point SC-FDE, the output signal vector can be given as [13]

$$
\mathbf{r}=F_{Q}^{H} H^{H} W H F_{Q} \Omega_{Q, L} \Upsilon_{L} \Gamma_{L, M} F_{M}^{H} T_{M, K} F_{K} \mathbf{a}+F_{Q}^{H} H^{H} W F_{Q} \mathbf{z}
$$

Then, the post-processing SINR can be given by

$$
\begin{aligned}
\operatorname{SINR}= & \frac{E_{s}}{\sigma_{n}^{2}+\sigma_{I S I}^{2}+\sigma_{\text {ITSI }}^{2}} \\
= & \frac{\left|\frac{1}{K} \sum_{k=0}^{K-1} \tilde{H}_{k}\right|^{2}}{\frac{\sigma^{2}}{K} \sum_{k=0}^{K-1} \bar{H}_{k}+\frac{1}{K} \sum_{k=0}^{K-1}\left|\tilde{H}_{k}\right|^{2}-\left|\frac{1}{K} \sum_{k=0}^{K-1} \tilde{H}_{k}\right|^{2}+\frac{1}{K} \sum_{d=1}^{D-1} \sum_{k=0}^{K-1}\left|\breve{H}_{d ; k}\right|^{2}}
\end{aligned}
$$

where $\sigma_{n}^{2}$ is variance of AWGN on demodulated symbols. $\sigma_{I S I}^{2}$ is the variance of the intersymbol-interference (ISI) within the $d$-th IFBT symbol, and can be expressed as $\sigma_{I S I}^{2}=\sum_{k=1}^{K-1}\left|h_{k}\right|^{2}=\frac{1}{K} \sum_{k=0}^{K-1}\left|\tilde{H}_{k}\right|^{2}-\left|\frac{1}{K} \sum_{k=0}^{K-1} \tilde{H}_{k}\right|^{2} \quad \cdot \quad \sigma_{I T S I}^{2}$ is the variance of the inter-IFBT symbol interference (ITSI) on the demodulated symbols, and can be expressed as $\sigma_{\text {ITSI }}^{2}=\frac{1}{K} \sum_{d=1}^{D-1} \sum_{k=0}^{K-1}\left|\breve{H}_{d ; k}\right|^{2}$. 


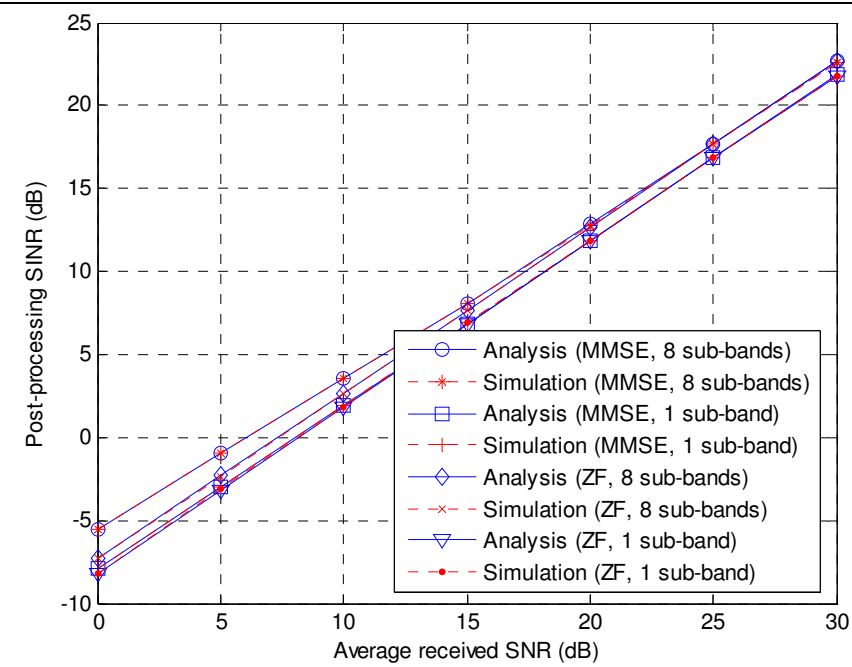

Fig. 19. The post-processing SINR of DFT-S-GMC with SC-FDE

Both the theoretical and simulated post-processing SINR are shown in Fig.19 for the DFT-SGMC receiver with MMSE and ZF SC-FDE respectively. Over the same channel condition, with MMSE equalization, DFT-S-GMC receiver achieves higher SINR than that with ZF equalization in the low SNR range and with wider band transmission, due to the noise enhancement effects of ZF SC-FDE.

b. Performance of frequency-domain implemented DFT-S-GMC transceiver

\begin{tabular}{|l|c|}
\hline System Parameters & Value \\
\hline Carrier frequency (GHz) & 2 \\
\hline Carrier Bandwidth (MHz) & 5 \\
\hline Sampling frequency (MHz) & 5.6 \\
\hline \# of total sub-bands (M) & 28 \\
\hline \# of useful sub-bands & 24 \\
\hline Upsampling rate & 32 \\
\hline \# of IFBT symbols in each data block (D) & 16 \\
\hline Sub-band BW (kHz) & 200 \\
\hline Sub-band 3dB-BW (kHz) & 175 \\
\hline Occupied BW (MHz) & 4.8 \\
\hline FFT size for FDE & 512 \\
\hline Prototype filter type & SRRC \\
\hline Simulation parameters & PB (3km/h) \\
\hline Channel model & Turbo (1/2) \\
\hline Channel coding(coding rate) & QPSK \\
\hline Modulation & MMSE \\
\hline Equalization & $1 / 1$ \\
\hline \# of Tx/Rx antennas & \\
\hline
\end{tabular}

Table 1. Simulation specification 
The simulation specification is shown in Table 1. As shown in the Fig.20, the BER performance of frequency-domain implemented DFT-S-GMC transceiver is almost the same as that of time-domain implemented DFT-S-GMC transceiver.

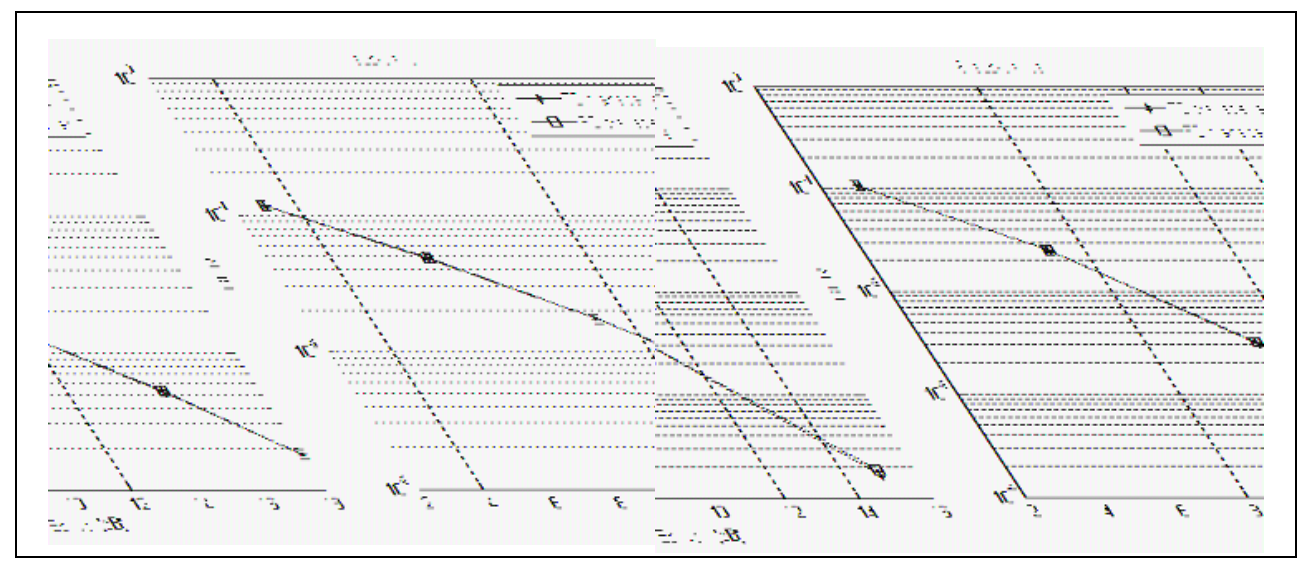

Fig. 20. BER performance comparison of FD transceiver with TD transceiver

As presented in the Table 2, the CM performance of frequency-domain implemented DFT-SGMC transmitter is very close to that of time-domain implemented DFT-S-GMC transmitter. Moreover, the CM of DFT-S-GMC is smaller 1.7 and 1.1dB than that of OFDM for QPSK and 16QAM modulation respectively.

\begin{tabular}{|c|c|c|c|c|c|c|}
\hline & \multicolumn{4}{|c|}{ Systems } \\
\hline & \multicolumn{2}{|c|}{$\begin{array}{c}\text { Frequency-domain } \\
\text { DFT-SMC }\end{array}$} & $\begin{array}{c}\text { Time-domain } \\
\text { DFT-S-GMC }\end{array}$ & \multicolumn{2}{c|}{ OFDM } \\
\hline $\begin{array}{c}\text { Used sub-carriers } \\
\text { / sub-band(s) }\end{array}$ & QPSK & 16QAM & QPSK & 16QAM & QPSK & 16QAM \\
\hline $16 / 1$ & 0.5 & 1.5 & 0.5 & 1.5 & 3.2 & 3.2 \\
\hline $32 / 2$ & 1.4 & 2.0 & 1.3 & 2.0 & 3.3 & 3.3 \\
\hline $64 / 4$ & 1.6 & 2.2 & 1.6 & 2.2 & 3.4 & 3.4 \\
\hline $256 / 16$ & 1.7 & 2.3 & 1.7 & 2.3 & 3.4 & 3.4 \\
\hline
\end{tabular}

Table 2. Cubic Metric (dB) comparison of frequency- and time-domain implemented DFT-SGMC system with OFDM system

\begin{tabular}{|c|c|c|c|}
\hline $\begin{array}{c}\text { \# of used } \\
\text { sub-band(s) }\end{array}$ & \multicolumn{2}{|c|}{$\begin{array}{c}\text { Computation complexity } \\
\text { (\# of real multiplications) }\end{array}$} & $\begin{array}{c}\text { Percentage of } \\
\text { reduction }\end{array}$ \\
\cline { 2 - 3 } & $\begin{array}{c}\text { TD transmitter/ } \\
\text { receiver }\end{array}$ & $\begin{array}{c}\text { FD transmitter/ } \\
\text { receiver }\end{array}$ & \\
\hline 28 & $18800 / 58688$ & $15184 / 31008$ & $19 \% / 47 \%$ \\
\hline 1 & $18432 / 55404$ & $9416 / 18868$ & $49 \% / 66 \%$ \\
\hline
\end{tabular}

Table 3. Complexity comparison of frequency- and time-domain implemented DFT-S-GMC transceiver 
As shown in the Table 3, with frequency-domain implementation structure, the computation complexity of DFT-S-GMC transceiver with equalization can be reduced significantly, compared with that with time-domain implementation structure. For 28 and 1 sub-band(s) transmission, the computational complexity can be reduced about $47 \%$ to $66 \%$.

\section{Conclusion}

In this chapter, the principle, implementation structure, time-frequency property of three Fourier Transform-based transmission systems, namely OFDM, DFT-S-OFDM and DFT-S$\mathrm{GMC}$, are presented for broadband wireless communications. For OFDM systems, the spectrum of each sub-carrier has a sinc-function shape, spectrums of all sub-carriers are independent each other which cause high PAPR of transmitted signal; For DFT-S-OFDM systems, each sub-carrier contains only a part of spectrum component of transmitted constellation symbols, and the time-domain waveform can be viewed as a DFT-based interpolation of transmitted constellation symbols, which bring in lower PAPR of transmitted signal; For DFT-S-GMC systems, each DFT-S-GMC symbol is formed by cyclically accumulating IFBT symbols with SRRC waveform in the time domain, hence, the spectrum of each sub-band has a Raised Cosine function shape, and due to DFT based spreading among sub-bands, the transmitted signal over all occupied sub-bands can be viewed as single-carrier signal as a whole. Moreover, the effects of time and frequency offset on OFDM and DFT-S-OFDM systems are analyzed quantitatively. Theoretical analysis and simulation results show that except the first symbol, all other demodulated symbols of DFTS-OFDM system have a better SIR than that of OFDM system under the same CFO condition. Furthermore, the post-processing SINR of DFT-S-OFDM and DFT-S-GMC are addressed for different equalizer. The closed-from expressions of SINR are presented and verified by the simulation results.

\section{References}

[1] S,B; Weinstein and Ebert, P.( 1971). Data transmission by frequency-division multiplexing using the discrete Fourier transform. IEEE Transactions on Communications, Vol. 19, No.5, (Oct. 1971) 628-634, ISSN: 0018-9332

[2] Myung, H.; Lim, J.; and Goodman, D.(2006). Single Carrier FDMA for Uplink Wireless Transmission. IEEE Vehicular Technology Magazine, Vol. 1, No. 3, (Sep. 2006) 30-38, ISSN: 1556-6072

[3] Cherubini, G.; Eleftheriou, E.; Cioffi, J.; Oker, S.(2000). Filter bank modulation techniques for very high speed digital subscriber lines. IEEE Communications Magazine, Vol. 38 , No. 5, (May 2000) 98-104, ISSN: 0163-6804

[4] Qiu, RC.; Liu, H.; Shen, X. (2005). Ultra-Wideband For Multiple Access Communications. IEEE Communications Magazine, Vol. 43, No.2, (February 2005) 80-87, ISSN: 01636804

[5] 3GPP TS 36.211 v8.7.0. Evolved Universal Terrestrial Radio Access (E-UTRA); Physical Channels and Modulation(Release 8), May 2009.

[6] 3GPP TR 36.913 V8.0.1 (2009-03) 3rd Generation Partnership Project, Technical Specification Group Radio Access Network, Requirements for further advancements for Evolved Universal Terrestrial Radio Access (E-UTRA) (LTE-A) (Release 8). 
[7] Yoon, D. and Cho, K.(2002) . On the general BER expression of one- and two dimensional amplitude modulations. IEEE Transactions on Communications, Vol. 50, No.7, (July 2002) 1074-1080, ISSN: 0018-9332

[8]Liu, Q.; Zhou,S.; and Giannakis, G.(2004). Cross-layer combining of adaptive modulation and coding with truncated ARQ over wireless links. IEEE Transactions on Wireless Communication., Vol. 3, No.5, (sept. 2004) 1746-755, ISSN: 1536-1276

[9] 3GPP TSG-RAN1 WG1 \\#36, R1-040189, New results on realistic OFDM interference, Malaga, Spain, Feb. 16-20, 2004.

[10] M, Li., Y, Rui. (2009). Analysis of CFO effects on and phase compensation method for SC-FDMA systems, Science in China Series F: Information Sciences, Vol.52, No. 12, (Dec. 2009) 2397-2405, ISSN: 1674-733X

[11] 3GPP TSG-RAN1 WG1 \\#42, R1-050719, Simulation Methodology for EUTRA UL: IFDMA and DFT Spread-OFDMA, London, U.K., Aug. 29 - Sept. 2, 2005.

[12] Zhang, X. ; Li, M.; Hu, H.; Wang, H.; Zhou, B.; You, X.(2006). DFT Spread Generalized Multi-Carrier Scheme for Broadband Mobile, IEEE PIMRC Communications. pp. 1-5, ISBN: 1-4244-0329-4, Helsinki , Sept. 2006.

[13] Li, M. ; Zhang, X.(2009). Performance analysis of DFT spread generalized multi-carrier Systems, Science in China Series F: Information Sciences, Vol.52, No.12, (Dec. 2009) 2385-2396, ISSN: 1674-733X

[14] Liu, P.; Bar-Ness, Y.(2006). Comparing the effect of Carrier Frequency Offset on OFDM and Single-Carrier Block Transmission in AWGN Channels, in Proc IEEE GLOBECOM 2006, pp. 1-5, ISBN: 1930-529X, San Francisco, Nov. 2006.

[15] Moose, Paul H.(1994). A technique for orthogonal frequency division multiplexing frequency offset correction, IEEE Transactions on communications, Vol.42, No.10, (Oct. 1994) 2908-2914, ISSN: 0090-6778

[16] Rui, Y.; Hu, H.; Li, M.; et al.(2009). Comparing Effects of Carrier Frequency Offset on Generalized Multi-carrier and OFDM Systems, in Proc IEEE ICC 2009, pp. 1-6, ISBN: 1938-1883, Dresden, Jun. 2009 


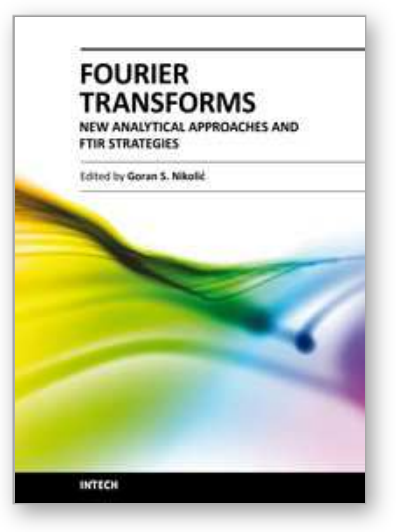

\author{
Fourier Transforms - New Analytical Approaches and FTIR \\ Strategies \\ Edited by Prof. Goran Nikolic
}

ISBN 978-953-307-232-6

Hard cover, 520 pages

Publisher InTech

Published online 01, April, 2011

Published in print edition April, 2011

New analytical strategies and techniques are necessary to meet requirements of modern technologies and new materials. In this sense, this book provides a thorough review of current analytical approaches, industrial practices, and strategies in Fourier transform application.

\title{
How to reference
}

In order to correctly reference this scholarly work, feel free to copy and paste the following:

Mingqi Li, Yun Rui and Zhiyong Bu (2011). Fourier Transform Based Transmission Systems for Broadband Wireless Communications, Fourier Transforms - New Analytical Approaches and FTIR Strategies, Prof. Goran Nikolic (Ed.), ISBN: 978-953-307-232-6, InTech, Available from: http://www.intechopen.com/books/fouriertransforms-new-analytical-approaches-and-ftir-strategies/fourier-transform-based-transmission-systems-forbroadband-wireless-communications

\section{INTECH}

open science | open minds

\author{
InTech Europe \\ University Campus STeP Ri \\ Slavka Krautzeka 83/A \\ 51000 Rijeka, Croatia \\ Phone: +385 (51) 770447 \\ Fax: +385 (51) 686166 \\ www.intechopen.com
}

\author{
InTech China \\ Unit 405, Office Block, Hotel Equatorial Shanghai \\ No.65, Yan An Road (West), Shanghai, 200040, China \\ 中国上海市延安西路65号上海国际贵都大饭店办公楼405单元 \\ Phone: +86-21-62489820 \\ Fax: $+86-21-62489821$
}


(C) 2011 The Author(s). Licensee IntechOpen. This chapter is distributed under the terms of the Creative Commons Attribution-NonCommercialShareAlike-3.0 License, which permits use, distribution and reproduction for non-commercial purposes, provided the original is properly cited and derivative works building on this content are distributed under the same license. 